\title{
An Efficient cDNA-AFLP-Based Strategy for the Identification of Putative Pathogenicity Factors from the Potato Cyst Nematode Globodera rostochiensis
}

\author{
Ling Qin, ${ }^{1}$ Hein Overmars, ${ }^{1}$ Johannes Helder, ${ }^{1}$ Herman Popeijus, ${ }^{2}$ Jeroen Rouppe van der Voort, ${ }^{1}$ \\ Wouter Groenink, ${ }^{1}$ Paul van Koert, ${ }^{1}$ Arjen Schots, ${ }^{2}$ Jaap Bakker, ${ }^{1}$ and Geert Smant ${ }^{1}$ \\ ${ }^{1}$ The Graduate School for Experimental Plant Sciences, Laboratory of Nematology, Wageningen University \\ and Research Center, Binnenhaven 10, 6709 PD Wageningen, The Netherlands; 'Laboratory of \\ Monoclonal Antibodies, Wageningen University and Research Center, Binnenhaven 10, \\ 6709 PD Wageningen, The Netherlands \\ Accepted 2 May 2000.
}

\begin{abstract}
A new strategy has been designed to identify putative pathogenicity factors from the dorsal or subventral esophageal glands of the potato cyst nematode Globodera rostochiensis. Three independent criteria were used for selection. First, genes of interest should predominantly be expressed in infective second-stage juveniles, and not, or to a far lesser extent, in younger developmental stages. For this, gene expression profiles from five different developmental stages were generated with cDNA-AFLP (amplified fragment length polymorphism). Secondly, the mRNA corresponding to such a putative pathogenicity factor should predominantly be present in the esophageal glands of pre-parasitic juveniles. This was checked by in situ hybridization. As a third criterion, these proteinaceous factors should be preceded by a signal peptide for secretion. Expression profiles of more than 4,000 genes were generated and three up-regulated, dorsal gland-specific proteins preceded by signal peptide for secretion were identified. No dorsal gland genes have been cloned before from plantparasitic nematodes. The partial sequence of these three factors, A4, A18, and A41, showed no significant homology to any known gene. Their presence in the dorsal glands of infective juveniles suggests that these proteins could be involved in feeding cell initiation, and not in migration in the plant root or in protection against plant defense responses. Finally, the applicability of this new strategy in other plant-microbe interactions is discussed.
\end{abstract}

Co-evolution of plants and microbes may result in intimate and durable interactions. A well-characterized example of coevolution of plants and microbes is the symbiosis of legumes and Rhizobia. Symbiosis arises from an extensive exchange of

Corresponding author: Ling Qin; Telephone: +31 317 485255; Fax +31 317 485267; E-mail: Ling.Qin@medew.nema.wau.nl

Current address of Jeroen Rouppe van der Voort: Keygene N. V., Agro Business Park 90, P.O. Box 216,6700 AE Wageningen, The Netherlands.

Nucleotide and/or amino acid sequence data are available in the EMBL, GenBank, and DDJB data bases under accession numbers AJ251756, AJ251757, and AJ251758. molecular signals between two partners. In contrast to Rhizobia, biotrophic bacteria, fungi, and nematodes often flourish at the expense of the hosting plant and release pathogenicity factors in order to reach a feeding site, counteract plant defense responses, and extract food.

In the case of sedentary, plant-parasitic nematodes, migration through plant tissues is facilitated by the secretion of $\beta$ 1,4-endoglucanases (Smant et al. 1998; Rosso et al. 1999). Apart from mechanical barriers, nematodes have to withstand the defense response of a host plant. Antioxidant enzymes in animal-parasitic nematodes are pivotal in the interaction with their host and, recently, the potato cyst nematode Globodera rostochiensis was shown to secrete superoxide dismutase upon exposure to 5-methoxy- $N, N$-dimethyltryptamine-hydrogen-oxalate (Robertson et al. 1999). To finally extract nutrients from its host, sedentary nematodes secrete molecules that force plant cells to re-differentiate into a feeding site. This process is marked by a reactivation of the cell cycle (Niebel et al. 1996). Typically, cells in a feeding site have large nuclei and show signs of enhanced metabolic activity. Nematode secretions have long been implicated as the causal agents of the physiological and molecular changes in feeding sites induced in plants (reviewed by Williamson and Hussey 1996). Recently, a protein fraction in nematode secretions smaller than 3 $\mathrm{kDa}$ was found to contain mitogenic activity on plant protoplasts and mammalian T-cell lymphocytes (Goverse et al. 1999). At present it is unclear whether this peptide fraction contains the causal agent(s) of feeding site induction.

The collection of pure nematode secretions essentially free from other microbial contaminants for protein analysis is a formidable challenge. Infective juveniles are very small and, because of their obligatory plant-parasitic nature and relatively long life cycle, it is difficult to rear them on a large scale in monoxenic cultures. The inability to collect sufficient nematode secretions for proper analysis prompted many groups in the past to develop polyclonal and monoclonal antibodies against nematode secretions. Some of the antisera were used to immunopurify proteins for sequence analysis (Ray et al. 1994; Smant et al. 1998). Although this laborious procedure has resulted in the identification of a pathogenicity factor in secretions of cyst nematodes (Smant 
et al. 1998; Yan et al. 1998), a more efficient method is desirable.

cDNA-AFLP (amplified fragment length polymorphism) is a novel RNA fingerprinting technique to display differentially expressed genes (Bachem et al. 1996). cDNAs are digested by two restriction enzymes and oligonucleotide adapters are ligated to the resulting restriction fragments to generate template DNA for polymerase chain reaction (PCR). PCR primers complementary to the adapter sequences with additional selective nucleotides at the $3^{\prime}$ end allow for the amplification of a limited number of cDNA fragments. Unlike differential display methods that make use of small random primers (Liang and Pardee 1992), relatively high annealing temperatures can be used and, hence, cDNA-AFLP is more stringent and reproducible. In contrast to hybridization-based techniques, such as cDNA microarrays, cDNA-AFLP can distinguish between highly homologous genes from individual gene families. In addition, cDNA-AFLP does not need any pre-existing sequence information, which makes it an excellent tool to identify novel genes.

To select genes encoding pathogenicity factors that are present in nematode secretions from a pool of thousands of amplified cDNA fragments, the following criteria were applied. As a first selection criterion, we assumed that cDNA fragments encoding pathogenicity factors are up-regulated in an RNA pool isolated from freshly hatched, pre-parasitic, second-stage juveniles (J2) just prior the invasion of a host plant, compared with younger developmental stages. Second, it was assumed that pathogenicity factors are produced in the dorsal or in the subventral esophageal glands of the nematode. This latter criterion is justified by numerous microscopic observations

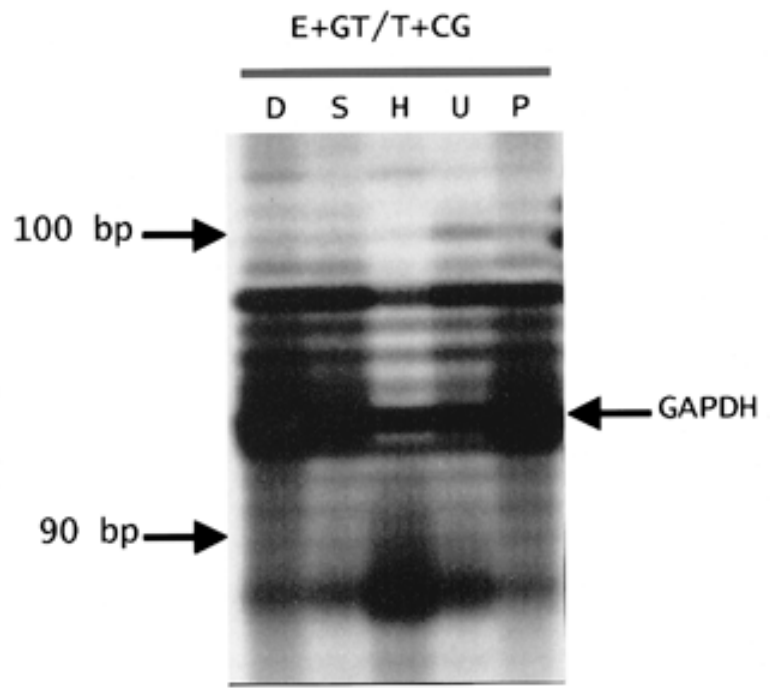

Fig. 1. Expression pattern of a glyceraldehyde-3-phosphate-dehydrogenase (GAPDH)-encoding mRNA from the potato cyst nematode Globodera rostochiensis as displayed by cDNA-amplified fragment length polymorphism (AFLP). Corresponding cDNA fragment was amplified with $E+G T / T+C G$. Band intensities were very similar in all five different developmental stages, which are indicated by capitals: D, unhatched second-stage juveniles (J2) in diapause; S, unhatched J2 after diapause, rehydrated for 2 days in water; $\mathrm{H}$, freshly hatched $\mathrm{J} 2$ in potato root diffusate; $\mathrm{U}$, developing nematodes (J1) in gravid females 2 months post inoculation; P, developing nematodes (J2) in gravid females 3 months post inoculation.
(Wyss and Zunke 1986; Wyss 1992). Third, we assumed that pathogenicity-related mRNAs encode a protein that starts with a signal peptide for secretion. This seems reasonable because pathogenicity factors act outside the nematode. The first pathogenicity factor cloned from cyst nematodes, a $\beta-1,4-$ endoglucanase, meets all of these criteria (Smant et al. 1998).

In this paper, the RNA fingerprints of approximately 4,000 genes in five distinct developmental stages from the potato cyst nematode $G$. rostochiensis were compared with cDNAAFLP. The life cycle of potato cyst nematodes allows for the isolation of highly synchronized, distinct phases. Amplified cDNA fragments up-regulated in infective $\mathrm{J} 2$ were sequenced and the $5^{\prime}$ ends of the cDNAs were checked for the presence of an open reading frame that starts with a predicted signal sequence for secretion. If such a signal was present, the exact location of this mRNA species was established by in situ hybridization. This highly efficient selection procedure resulted in the identification of three dorsal gland-specific genes. It is noted that, despite considerable efforts, to date no proteins have been identified from the dorsal glands of sedentary nematodes, and the three dorsal glands protein encoding cDNAs reported here are the first ones to be cloned.

\section{RESULTS}

Validation of the quantitative relationships of transcript-derived fragments in cDNA-AFLP.

The expression patterns of more than 4,000 transcriptderived fragments (TDFs) from five successive developmental stages of the potato cyst nematode G. rostochiensis were monitored by cDNA-AFLP analysis. The highly synchronous inoculation of potato plants allowed for a comparison between the mRNA populations of potato root diffusate (PRD)-hatched $\mathrm{J} 2$ and mRNA populations of younger stages: developing nematodes in young cysts (2 months post inoculation); developing nematodes in young cysts ( 3 months post inoculation); unhatched J2 in diapause; and rehydrated, unhatched J2 after diapause. In the first paper about cDNA-AFLP, the expression levels revealed by cDNA-AFLP were shown to be comparable with results obtained by Northern (RNA) blot analysis (Bachem et al. 1996). To establish whether the intensities of TDFs revealed by cDNA-AFLP correctly reflected the differences in the original mRNA populations in our system, the expression patterns of constitutively (glyceraldehyde-3phosphate-dehydrogenase [GAPDH], encoding cDNA named $g p d$ ) and transiently expressed genes (GR-engs) were monitored.

A cDNA encoding the enzyme GAPDH (Qin et al. 1998) was taken as a reference. The crucial role of GAPDH in glycolysis and gluconeogenesis suggests a constitutive expression of gpd in G. rostochiensis. If cDNA-AFLP correctly reflects the abundances of GAPDH-encoding mRNAs, similar band intensities would be observed in all five developmental stages. Based on sequence information, digestion of the GAPDH-encoding cDNA with EcoRI and TaqI would produce a band of $93 \mathrm{bp}$. Two selective primer extensions were used (E + GT, T+ CG, for "E" and "T" see Materials and Methods) to reduce the complexity and facilitate the identification of the proper TDF. In all stages, TDFs were observed at the predicted position and the intensities of the corresponding bands were nearly identical (Fig. 1). The 93- bp TDF was isolated 
from the gel, and sequencing revealed a perfect match with gpd.

$\beta$-1,4-endoglucanase shows a characteristic expression pattern in potato cyst nematodes with high levels of transcription just prior to the onset of parasitism (Smant et al. 1997). To date, two highly homologous $\beta$-1,4-endoglucanases (GR-eng1 and GR-eng-2) have been identified in G. rostochiensis (Smant et al. 1998). Based on available sequence information, a band of $398 \mathrm{bp}$ is expected to appear on a cDNA-AFLP gel. Having an ancillary cellulose binding domain, ENG-1 is substantially larger than ENG-2, and it includes the restriction sites for a second TDF (104 bp). The primer combination E+TT and T+TG was used to amplify the 398-bp fragment. The extensions E+AA and T+GG were employed for the 104bp fragment of ENG-1 (data not shown). The resulting AFLP patterns showed amplification products of both sizes. The highest band intensities were observed in infective $\mathrm{J} 2$ and in rehydrated, unhatched J2 (Fig. 2), which corresponds with our previous findings (Smant et al. 1997). The 398-bp TDF sequence showed $99 \%$ identity with GR-eng-2.

To further verify the reliability of this system, a semiquantitative PCR method was used to study the expression levels of $\beta$-1,4-endoglucanases in the five different life stages. Semiquantitative PCR was carried out on cDNAs made from these stages. The expression levels of $\beta$-1,4-endoglucanases were normalized by the levels of gpd expression. A very similar expression pattern was observed, compared with cDNA-AFLP (Fig. 3).

It is concluded that cDNA-AFLP correctly reflects the expression patterns of a constitutively expressed gene, gpd, and the developmentally regulated genes, GR-eng-1 and GR-eng-2.

\section{The identification of TDFs with a pathogenicity factor like expression pattern.}

Assuming that the kinetics of expression revealed by cDNA-AFLP indeed reflect the mRNA abundances in the original mRNA populations, eight primer combinations were used $(\mathrm{E}+\mathrm{A} / \mathrm{T}$ and $\mathrm{T}+\mathrm{A} / \mathrm{C} / \mathrm{G} / \mathrm{T})$ to generate a large number

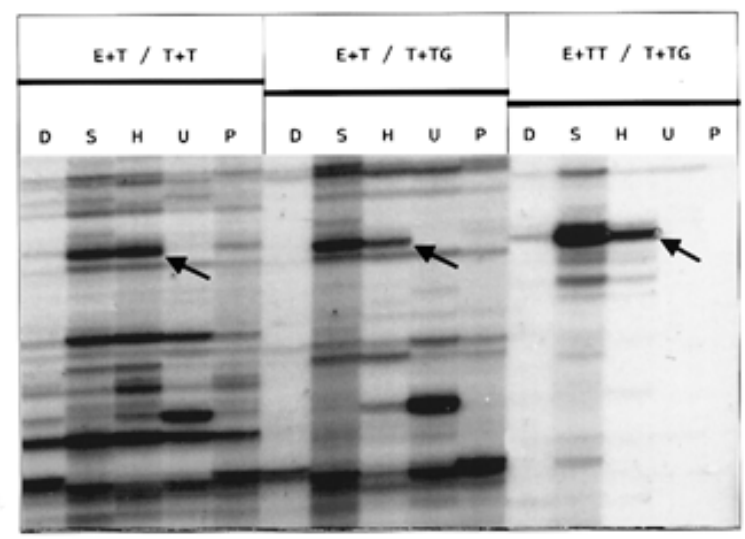

Fig. 2. The expression pattern of $\beta$-1,4-endoglucanases-encoding mRNAs from five different developmental stages of the potato cyst nematode Globodera rostochiensis as displayed by cDNA-amplified fragment length polymorphism (AFLP). Arrows point to bands produced by Gr-engs. Three different primer extensions were used: $\mathrm{E}+\mathrm{T} / \mathrm{T}+\mathrm{T}$, E+T/T+TG and E+TT/T+TG. D, S, H, U, and P refer to different developmental stages (for explanation, see Figure 1 caption). of mRNA expression profiles. Each primer combination resulted in about 230 discrete bands on large polyacrylamide gels. The complexity of the resulting patterns prompted us to use one additional selective nucleotide $(\mathrm{T}+2$ instead of $\mathrm{T}+1)$. Hence, E+C/G was used in combination with all possible $\mathrm{T}+2$ extensions and, as a consequence, the average number of TDFs dropped to 85 per lane. In total, 40 primer combinations were tested, resulting in the visualization of about 4,500 TDFs. Approximately 100 TDFs (approximately 2\%) appeared to be up-regulated in rehydrated cysts and/or in infective J2. A sample of 14 TDFs was excised from gel, cloned, and sequenced. To check whether the obtained sequences indeed corresponded with the up-regulated TDFs, one or two additional selective nucleotides were introduced complementary to the sequences at the $5^{\prime}$ and $3^{\prime}$ ends of the fragment. From the 14 TDFs tested, 10 TDFs still displayed the same expression pattern and only these TDFs were analyzed further. In a control experiment, the effect of additional selective nucleotides on the expression pattern of $\beta$-1,4-endoglucanases was verified. The expression patterns were nearly identical, irrespective of the number of selective nucleotides (Fig. 2). Except for one TDF (A13) that shows high homology with a 4-hydroxyphenylpyruvate dioxygenase gene (data not shown), no significant homologies with other known genes were found among the other up-regulated TDFs.

\section{In situ hybridization of up-regulated TDFs.}

Subventral and dorsal esophageal gland products are thought to be crucial in the interaction between endoparasitic nematodes and their host. This can be illustrated by $\beta-1,4-$-endoglucanase, the first pathogenicity factor cloned from a plant-parasitic nematode. In situ hybridization in pre-parasitic $\mathrm{J} 2$ of G. rostochiensis showed labeling in the subventral glands only (Smant et al. 1998). For $10 \mathrm{TDFs}$, sense and antisense DNA probes were made by asymmetric PCR with either the E+0 or the $\mathrm{T}+0$ primer. We have observed that the background staining with DNA probes was always much less than with RNA probes. Antisense DNA probes of the TDFs A4, A18, and A41 specifically labeled the dorsal gland of infective $\mathrm{J} 2$. Labeling was observed around the nucleus in the gland cell, and no hybridization was detectable in the gland cell extensions or the ampullae. No labeling was observed for the sense probes. The detected transcript levels of A4, A41, and A18 were respectively high, moderate, and low (Fig. 4). These three TDFs appeared to be upregulated in the PRD-hatched $\mathrm{J} 2$ stage and this expression pat-

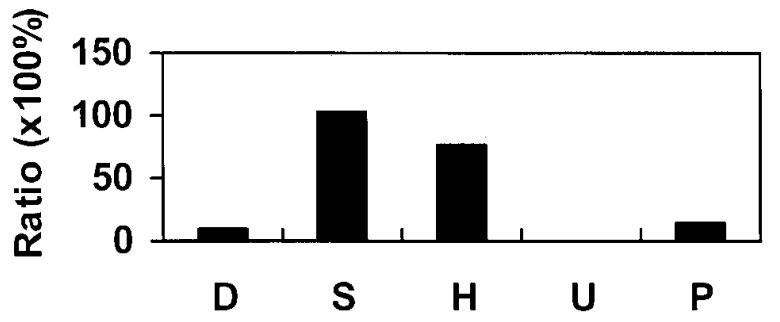

Fig. 3. Semiquantitative polymerase chain reaction (PCR) analysis of $\beta$ 1,4-endoglucanases expression in five different developmental stages of the potato cyst nematode Globodera rostochiensis. Y-axis: ratio is given between net intensities of $\beta$-1,4-endoglucanases and glyceraldehyde-3phosphate-dehydrogenase (GAPDH). D, S, H, U, and P refer to different developmental stages (for explanation, see Figure 1 caption). 
tern was confirmed with primers with corresponding selective extensions at the $3^{\prime}$ end (Fig. 5). The antisense probe of A11 labeled the rectal gland (data not shown). The remaining six probes did not show any hybridization signal.

\section{Analysis of $5^{\prime}$ ends of mRNAs possibly encoding nematode-secreted compounds.}

A dorsal gland protein could be a constituent of the nematode secretions if it is preceded by a signal peptide for secretion. To find out whether the up-regulated, dorsal glandspecific TDFs A4, A18, and A41 included such a signal, the $5^{\prime}$ end of the cDNAs was amplified from a cDNA library (Smant et al. 1998). For A4, a fragment of 162 bp was obtained showing an overlap of $59 \mathrm{bp}$ with the $5^{\prime}$ end of the corresponding TDF. Similarly, a 733-bp fragment was obtained for A18, which had $61 \mathrm{bp}$ in common with the $5^{\prime}$ end of the TDF sequence. For A41, a 470-bp fragment was isolated having $175 \mathrm{bp}$ in common with the $5^{\prime}$ end of the A41 TDF sequence. In all three cases, a putative start codon could be identified preceded by three to four adenine residues, a phenomenon frequently observed in $C$. elegans genes (Blumenthal et al. 1997) and also in potato cyst nematode genes (L. Qin, unpublished observations). The conceptual translation predicted that A4, A18, and A41 contained open reading frames. The computer algorithm SignalP (Nielsen et al. 1997) was used to assess whether the protein was preceded by a signal peptide for secretion. In all three cases, such a signal was predicted to be present. It is concluded that the proteins corre- sponding to the TDFs A4, A18, and A41 are produced in the dorsal gland and, most likely, secreted through the stylet.

\section{DISCUSSION}

In this paper, a novel, cDNA-AFLP-based strategy is described that resulted in the cloning of three putative pathogenicity factors-named A4, A18, and A41-from the dorsal esophageal gland of the potato cyst nematode G. rostochiensis. Secretions from the subventral and dorsal esophageal glands of cyst and root knot nematodes are thought to be essential for many aspects of parasitism (Williamson and Hussey 1996). The first cloned secretory product from the subventral glands of a cyst nematode was shown to be a $\beta$-1,4-endoglucanase (Smant et al. 1998). The three dorsal gland genes presented here are the first ones to be identified from a plant-parasitic nematode. None of these putative secretion compounds show significant homology to any known protein. Hence, the functions of A4, $\mathrm{A} 18$, and $\mathrm{A} 41$ in the interaction between the potato cyst nematode and its host plants remain to be established.

To identify proteinaceous pathogenicity factors present in the secretory glands of the potato cyst nematode, we developed a strategy that uses the corresponding mRNAs as a starting point. Pools of mRNAs of distinct developmental stages were compared by cDNA-AFLP. Using this PCR-based technology, we circumvented the problem of scarcity of starting material. Cyst nematodes have a single infective stagethe pre-parasitic $\mathrm{J} 2$ - and we postulate that pathogenicity fac-
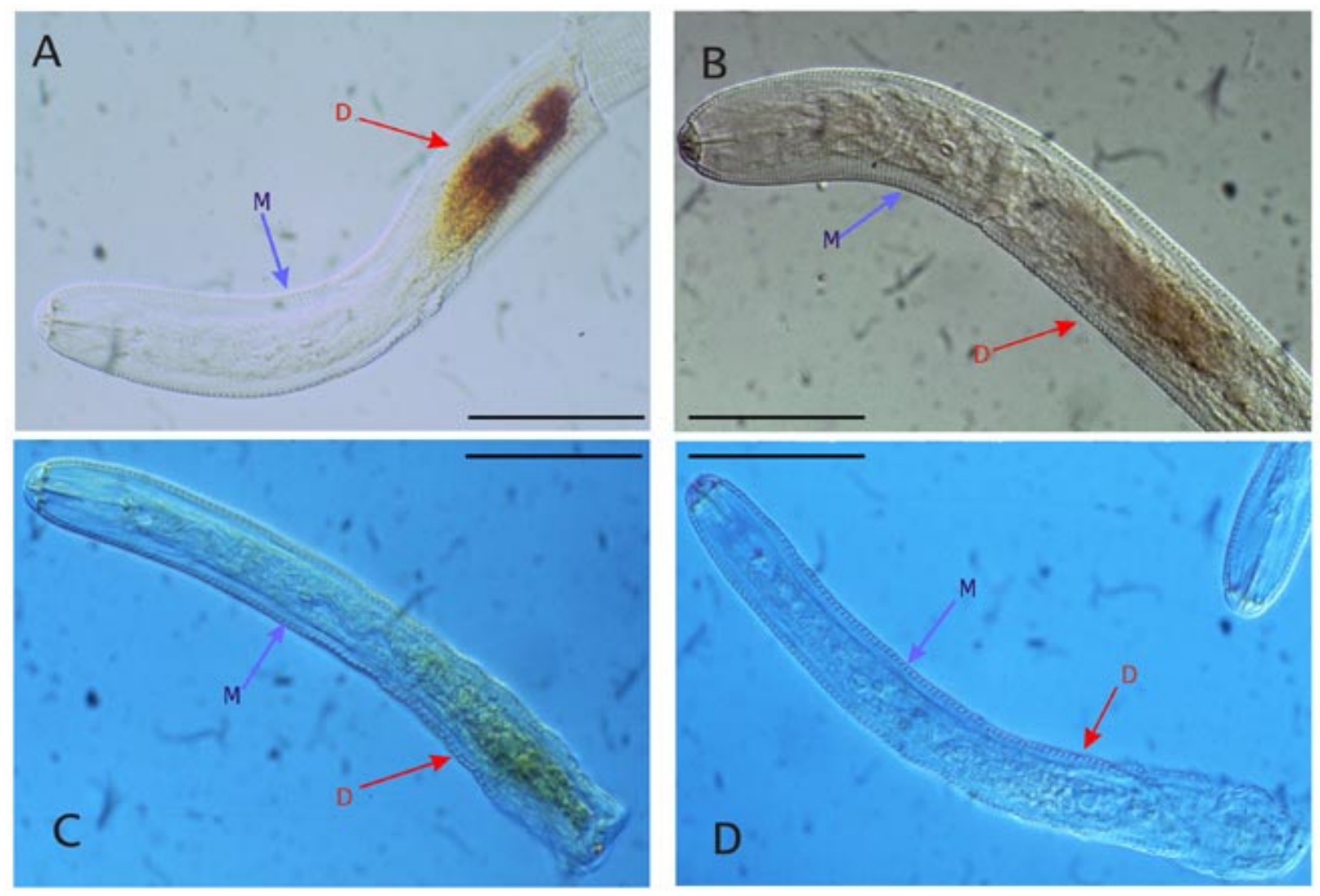

Fig. 4. In situ hybridization with three different transcript-derived fragment (TDF)-derived probes from Globodera rostochiensis. Labeling was done in pre-parasitic second-stage juveniles (J2). Labeling patterns with (A) A4, (B) A18, and (C) A41 antisense probes. D, Typical image of a sense probe labeling. Red arrows point at dorsal gland (D). Purple arrows point at metacorpus (M). Scale bar $=20 \mu \mathrm{m}$. 
tors related to early events in the infection process will predominantly be present in this life stage and they will be functional outside the nematode. Although the results presented in this paper show the usefulness of our selection criteria, it is noted that exceptions (e.g., a pathogenicity factor that is expressed constitutively) will be missed.

First, the reliability of the expression patterns as displayed by the cDNA-AFLP technique was verified. In contrast to differential display, in which random primers are used for amplification of cDNAs (Liang and Pardee 1992), cDNA-AFLP permits a relatively easy validation of the displayed profiles with control genes with known expression patterns. The cDNA fragment corresponding to the constitutively expressed gene gpd-1 was present in all developmental stages with similar intensities. The amplified fragment from GR-eng-1 was up-regulated in pre-parasitic stages, and this result corresponded well with a previous study of $\beta$-1,4-endoglucanases at the protein level (Smant et al. 1997). It is noted that the expression profiles of GR-eng-1 and -2 (95\% identical in the first 884 nucleotides) could be monitored separately. This represents a clear advantage of cDNA-AFLP technique over the hybridization-based methods, in which the hybridization signals from each of the highly homologous sequences will be indistinguishable. The validity of the expression patterns was further verified with additional selective nucleotides. By doing so, we drastically reduced the number of displayed amplification products and, as a consequence, the primer/target ratio was changed. This change did not affect the relative intensities of the control genes. In a previous study, gene expression during potato tuber formation as revealed by cDNA-AFLP

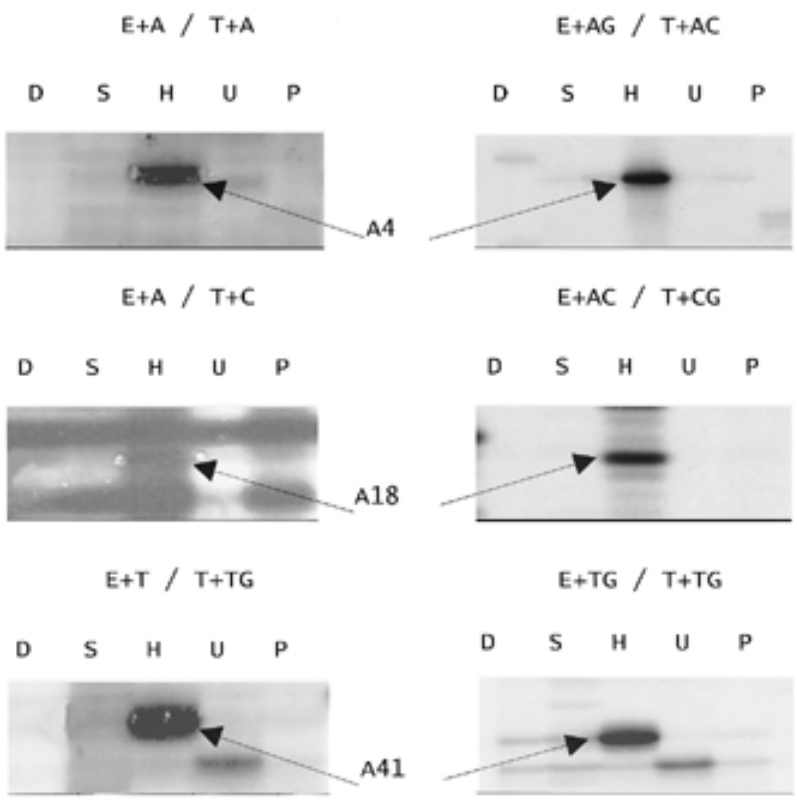

Fig. 5. Expression patterns of three transcript-derived fragments (TDFs), $\mathrm{A} 4, \mathrm{~A} 18$, and $\mathrm{A} 41$, from the potato cyst nematode Globodera rostochiensis as revealed by cDNA-amplified fragment length polymorphism (AFLP). Arrow-pointed bands from left panel were cut out, cloned, and sequenced. On the basis of the sequence results, primers $\mathrm{E}$ and $\mathrm{T}$ were extended with one or two additional selective nucleotides. Resulting cDNAAFLP expression patterns are shown (right panel). D, S, H, U, and P refer to different developmental stages (for explanation, see Figure 1 caption). was checked by Northern analysis and similar results were obtained (Bachem et al. 1996). It is concluded that, for the limited number of control genes available for G. rostochiensis, the correctness of the gene expression profiles as revealed by cDNA-AFLP could be confirmed.

In this paper, the expression levels of 4,560 TDFs in five developmental stages of $G$. rostochiensis were monitored. Certainly, a small number of transcripts will be represented by more than one TDF. In a similar study on potato tuber formation process, the authors found that about $2 \%$ out of the 200 cloned TDFs appeared to be derived from the same transcript (Bachem et al. 1996). Given the fact that this double representation could only be detected when both fragments hit upon the same gene sequence in the data base during homology search, we presume this percentage is an underestimation. If we suppose that $10 \%$ of the displayed TDFs are derived from the same transcript, the expression patterns of about 4,000 genes were displayed.

To estimate the efficiency of the cDNA-AFLP approach, it is useful to make a comparison with Caenorhabditis elegans. The $C$. elegans genome sequencing project revealed that the $97 \mathrm{Mb}$ haploid genome sequence of this free-living nematode contains 19,099 predicted protein-coding genes. Exon sequences are predicted to cover $27 \%$ of the genome, giving an average size of $1.35 \mathrm{~kb}$ per transcript (The C. elegans Sequencing Consortium 1998). The genome sizes of G. rostochiensis and C. elegans are similar (Rouppe van der Voort et al. 1999). Under the assumption that the average transcript size of $G$. rostochiensis is about $1.35 \mathrm{~kb}$ as well, approximately $33 \%$ of the potato cyst nematode genes will be cut at least once $\left(1.35 \mathrm{~kb} / 4^{6} \mathrm{bp}=33 \%\right)$ by EcoRI recognizing a 6-bp motive. The frequent cutter TaqI will restrict most of the cDNA fragments generated by EcoRI. If these assumptions are correct, the estimated number of expressed genes in juveniles of the potato cyst nematode is about $12,000(=4,000 /$ 0.33 ). If a second restriction enzyme combination is used, the coverage of the genome should reach about 56\% $(=33 \%+(1-$ $33 \%) \times 33 \%)$, a third combination about $70 \%(=56 \%+(1-$ $56 \%) \times 33 \%$ ). In fact, a survey of the expression patterns of the genes representing a significant part of the genome can be achieved with relatively small investment both in time and financial resources.

Among the 4,000 expression profiles, approximately 100 TDFs were up-regulated in rehydrated cysts and/or in infective J2. In situ hybridizations showed that three TDFs-A4, A18, and A41-were specifically produced in the dorsal gland in infective, pre-parasitic juveniles. The presence of a predicted signal peptide for secretion indicates that these proteins are translocated in the secretory pathway and, as such, they meet the criteria set out for putative pathogenicity factors of the potato cyst nematode. The partial cDNA sequences did not reveal a significant homology with known proteins. Their presence in the dorsal esophageal gland (and their absence in the subventral glands) suggests that these proteins could be involved in processes finally resulting in food extraction from the host plant, and presumably not in migration or in protection against host plant defense reactions.

Knock-out mutants would be an ideal tool to unravel the role of these dorsal gland proteins in pathogenicity. Introduction of double-stranded RNA has been shown to specifically disrupt the activity of genes containing homologous sequences 
in C. elegans (Fire et al. 1998). Alternatively, target-binding, single-chain antibodies could be expressed in the cytosol of cortical root cells. With this approach, the relative importance of a putative pathogenicity factor could be assessed (Schots et al. 1992). A third, more general approach is the heterologous expression of putative pathogenicity factors in plants. The resulting phenotypes could give an indication about the possible roles of these putative pathogenicity factors in parasitism.

This paper illustrates the efficiency of a novel strategy for the identification of pathogenicity factors from the potato cyst nematode $G$. rostochiensis. This relatively general approach can be applied to any plant-pathogen combination as long as distinct mRNA populations can be isolated from infectious and non-infectious life stages of the pathogen. This technique will be particularly suitable for many non-model organisms for which limited genome sequence information is available. In contrast to, for example, expressed sequence tags (ESTs), which rely solely on homology searches, this cDNA-AFLP-based approach allows for the identification of putative pathogenicity factors that are specifically expressed at infective stages, even if they do not show significant homology to any known gene. As such, this strategy could be a valuable tool in the identification of pathogen signals mediating host plant manipulation.

\section{MATERIALS AND METHODS}

\section{Nematode culture.}

G. rostochiensis pathotype Ro1-Mierenbos was cultured as described previously (de Boer et al. 1992). RNA was extracted from five developmental stages: ( $\mathrm{D}=$ dormant) dehydrated, unhatched, $\mathrm{J} 2$ in cysts (in diapause); ( $\mathrm{S}=$ soaked) rehydrated, unhatched $\mathbf{J} 2$ in 1-year-old cysts after exposure to sterile tap water for 2 days; $(\mathrm{H}=$ hatched) pre-parasitic $\mathrm{J} 2$ (dry cysts were incubated in sterile tap water for 1 week, tap water is replaced by potato root diffusate [PRD] in the second week); $(\mathrm{U}=$ undifferentiated into $\mathrm{J} 2)$ developing nematodes (mostly $\mathrm{J} 1$ ) in gravid females 2 months post inoculation; ( $\mathrm{P}=$ prediapause) developing nematodes (J2) in gravid females 3 months post inoculation.

\section{RNA isolation.}

From each of the five developmental stages about $100 \mu \mathrm{g}$ of total RNA was isolated with TRIzol Reagent (Life Technologies, Breda, The Netherlands). The integrity of the total RNA was checked on a denaturing agarose gel. Dynabeads Oligo $(\mathrm{dT})_{25}$ (Dynal A.S, Oslo, Norway) were used to isolate about 1 $\mu \mathrm{g}$ of mRNA from each of the developmental stages.

\section{cDNA-AFLP analysis.}

With some minor modifications, cDNA-AFLP was performed as described (Bachem et al. 1996). In short, cDNA was synthesized from $1 \mu \mathrm{g}$ of mRNA with the Superscript Choice System for cDNA synthesis-kit (Life Technologies). The first-strand cDNA was reverse transcribed with a $\mathrm{T}_{12-18}$ oligonucleotide primer. Subsequently, cDNA was digested by restriction endonucleases EcoRI and TaqI and ligated to EcoRI and TaqI adapters (Vos et al. 1995). Pre-amplification was performed in 25 cycles with primers (indicated as "E" and "T") corresponding to the EcoRI and TaqI adapters without extension (E+0: GAC TGC GTA CCA ATT C and T+0: GAT GAG TCC TGA CCG A) with a standard pre- amplification program. After $100 \times$ dilution of the PCR product, the template was amplified again with one or two selective base extensions at the $3^{\prime}$ end of the primers $\mathrm{E}$ and $\mathrm{T}$ with a standard AFLP touchdown-selective amplification program. As fragment size references on gel $(50 \mathrm{~cm}$ in length), a 30 - to 330-bp AFLP ladder (Life Technologies) was used in combination with a 50- to 500-bp SequaMark ladder (Research Genetics, Huntsville, Canada). Differentially expressed bands were cut out from the gel and re-amplified with High Fidelity polymerase (Roche Diagnostics, Mannheim, Germany). The amplified fragments were checked on a $2 \%$ agarose gel and cloned subsequently into pCR2.1-TOPO vector (Invitrogen, Leek, the Netherlands). Transformed Escherichia coli colonies were checked by PCR with the same primers used in the selective amplification to confirm the presence of the expected insert. Plasmids were purified with the Wizard Plus Miniprep DNA Purification System (Promega, Madison, WI) and sequenced on either an Automated Laser Fluorescent DNA sequencer (Pharmacia, Uppsala, Sweden) or a LI-COR DNA sequencer (LI-COR, Lincoln, NE). Confirmation of the cloned cDNA-AFLP fragments was done with one additional selective base to the $3^{\prime}$ ends of the $\mathrm{E}$ and/or T primers in selective amplifications and run on gel.

\section{Semiquantitative PCR.}

To validate the cDNA-AFLP expression patterns, semiquantitative RT-PCR was performed on $\beta$-1,4-endoglucanases and GAPDH encoding mRNAs from five different stages. $\beta-1,4-$ endoglucanases are pathogenicity factors of $G$. rostochiensis and these cell wall-degrading enzymes are abundantly expressed in J2 within cysts soaked with water and PRDhatched J2 (Smant et al. 1997). The primers cel-up (5'TGGAACGCCGATACAGTGAAGG-3') and cel-down (5',GGAGGCGTAGAAGTGGAAGGTGTA-3') were used to amplify both endoglucanases (GR-eng-1 and GR-eng-2). cDNA was prepared as described above from $1 \mu \mathrm{g}$ of mRNA. A small fraction $(0.6 \%)$ of synthesized cDNA was used as template in a PCR. The primers gpd-up (5'-GCGCTGCGGT TGAGAAGGAC-3') and gpd-down (5'-CCGGTGCTGGCA GGGATGATGT-3') were used to amplify the corresponding cDNA. PCR was performed in a $100-\mu l$ reaction volume containing $1 \times$ Supertaq PCR buffer (SphaeroQ, Breda, The Netherlands), $0.8 \mathrm{mM}$ dNTPs, $0.2 \mathrm{U}$ Supertaq, and $0.25 \mu \mathrm{M}$ concentrations of each primer. A touchdown PCR profile was used: 5 cycles of $20 \mathrm{~s}$ at $92^{\circ} \mathrm{C}, 30 \mathrm{~s}$ at $65^{\circ} \mathrm{C}, 60 \mathrm{~s}$ at $72^{\circ} \mathrm{C}$, followed by 29 cycles of $20 \mathrm{~s}$ at $92^{\circ} \mathrm{C}, 30 \mathrm{~s}$ at $60^{\circ} \mathrm{C}, 60 \mathrm{~s}$ at $72^{\circ} \mathrm{C}$. A $10-\mu \mathrm{l}$ aliquot was taken out every three cycles and analyzed on a $2 \%$ agarose gel including $0.4 \mu \mathrm{g}$ of ethidium bromide per $\mathrm{ml}$. In the log-linear phase of the amplification reaction, the PCR products were analyzed with KODAK 1D image analysis software (Eastman Kodak, Rochester, NY). The amount of PCR product corresponding to $\beta$-1,4-endoglucanases was normalized with GAPDH as baseline reference.

\section{Sequence analysis.}

DNA sequences were analyzed with the Laser gene software package (DNASTAR, Madison, WI). Data base search was done with WU-Blast at the Human Genome Center, Baylor College of Medicine, BLAST service at the National Center for Biotechnology Information (NCBI), and FASTA at European Bioinformatics Institute (EBI). The computer algo- 
rithm SignalP (Nielsen et al. 1997) was used to predict the presence of a signal peptide for secretion and the corresponding putative cleavage site.

\section{In situ hybridization.}

Except for the use of single-stranded DNA (instead of RNA) probes-synthesized by linear PCR as described (Tabara et al. 1996) - the nematode fixation, hybridization, and detection steps were essentially performed as described (de Boer et al. 1999). Briefly, cDNA-AFLP fragments cloned in pCR2.1-TOPO vector (Invitrogen) were first amplified with Universal M13 (-20) forward and Universal M13 reverse primers (Invitrogen). The vector sequence from the amplified product was removed by the addition of EcoRI $\left(37^{\circ} \mathrm{C}, 1 \mathrm{~h}\right)$. The digested product was checked on $1 \%$ agarose gel. The sense and antisense probes were made by asymmetric PCR in the presence of digoxigenin (DIG)-dUTP (Roche Diagnostics) with either the $\mathrm{E}+0$ or $\mathrm{T}+0$ primer with $20 \mathrm{ng}$ of digested product as template in a $20-\mu 1$ reaction volume. The DIGlabeled probes were purified through a G50 Mini Quick Spin DNA column (Roche Diagnostics) and $10 \mu \mathrm{l}$ of Tris-EDTA (TE) buffer was added. In each hybridization reaction, $30 \mu \mathrm{l}$ of labeled probe was used. Alkaline phosphatase activity was detected by the addition of X-phosphate and NBT (Roche Diagnostics), and labeled infective juveniles were observed under a Leica inverted microscope (Leica, Deerfield, IL).

\section{Cloning of the 5 ' end of the cDNA.}

Gene-specific primers were used in combination with vector primers in pcDNAII to amplify the $5^{\prime}$-end region of each cDNA-AFLP fragment (A4, A18, A41) from a cDNA library constructed in pcDNAII as described (Smant et al. 1998).

\section{ACKNOWLEDGMENTS}

We wish to thank Christian Bachem and Peter van Zandvoort (Wageningen University and Research Center) for very useful discussions concerning the cDNA-AFLP technique. We would like to extend our gratitude to Jan de Boer and Thomas Baum (Iowa State University, U.S.A.) for introducing us to the in situ hybridization technique and also Tom Tytgat and Godelieve Gheysen (University of Gent, Belgium) for useful discussion about in situ protocols. We are grateful to Andrew Gane (Cardiff University, U.K.) for sequencing some of the clones. This work was supported by EC grant BIO4-CT96-0318 (LQ).

\section{LITERATURE CITED}

Bachem, C. W. B., Van Der Hoeven, R. S., De Bruijn, S. M., Vreugdenhil, D., Zabeau, M., and Visser, R. G. 1996. Visualization of differential gene expression using a novel method of RNA fingerprinting based on AFLP: Analysis of gene expression during potato tuber development. Plant J. 9:745-753.

Blumenthal, T., and Steward, K. 1997. RNA processing and gene structure. Pages 117-146 in: C. elegans II. D. L. Riddle, T. Blumenthal, B. J. Meyer, and J. R. Priess, eds. Cold Spring Harbor Laboratory, Cold Spring Harbor, NY.

The $C$. elegans Sequencing Consortium. 1998. Genome sequence of the nematode $C$. elegans: A platform for investigating biology. Science 282:2012-2018.

de Boer, J.M., Overmars, H. A., Bakker, J., and Gommers, F. J. 1992. Analysis of two-dimensional protein patterns from developmental stages of the potato cyst nematode, Globodera rostochiensis. Parasitology 105:461-474.

de Boer, J.M., Yan, Y., Wang, X., Smant, G., Hussey, R. S., Davis, E. L., and Baum, T. J. 1999. Developmental expression of secretory $\beta-1,4-$ endoglucanases in the subventral esophageal glands of Heterodera glycines. Mol. Plant-Microbe Interact. 12:663-669.

Fire, A., Xu, S., Montgomery, M. K., Kostas, S. A., Driver, S. E., and Mello, C. C. 1998. Potent and specific genetic interference by doublestranded RNA in Caenorhabditis elegans. Nature 391:806-811.

Goverse, A., Rouppe van der Voort, J., Rouppe van der Voort, C., Kavelaars, A., Smant, G., Schots, A., Bakker, J., and Helder, J. 1999. Naturally induced secretions of the potato cyst nematode co-stimulate the proliferation of both tobacco leaf protoplasts and human peripheral blood mononuclear cells. Mol. Plant-Microbe Interact. 12: 872-881.

Liang, P., and Pardee, A. B. 1992. Differential display of eukaryotic messenger RNA by means of the polymerase chain reaction. Science 257:967-971.

Niebel, A., Engler, J. D., Hemerly, A., Ferreira, P., Inze, D., Vanmontagu, M., and Gheysen, G. 1996. Induction of cdc2a and cyc1at expression in Arabidopsis thaliana during early phases of nematode induced feeding cell formation. Plant J. 10:1037-1043.

Nielsen, H., Engelbrecht, J., Brunak, S., and Von Heijne, G. 1997. Identification of prokaryotic and eukaryotic signal peptide and prediction of their cleavage sites. Protein Eng. 10:1-6.

Qin, L., Smant, G., Stokkermans, J., Bakker, J., Schots, A., and Helder, J. 1998. Cloning of a trans-spliced glyceraldehyde-3-phosphatedehydrogenase gene from the potato cyst nematode Globodera rostochiensis and expression of its putative promoter region in Caenorhabditis elegans. Mol. Biochem. Parasitol. 96:59-67.

Ray, C., Abbott, A. G., and Hussey, R. S. 1994. trans-splicing of a Meloidogyne incognita mRNA encoding a putative esophageal gland protein. Mol. Biochem. Parasitol. 68:93-101.

Robertson, L., Robertson, W. M., and Jones, J. T. 1999. Direct analysis of the secretions of the potato cyst nematode Globodera rostochiensis. Parasitology 119:167-176.

Rosso, M.-N., Favery, B., Piotte, C., Arthaud, L., de Boer, J. M., Hussey, R. S., Bakker, J., Baum, T. J., and Abad, P. 1999. Isolation of a cDNA encoding a $\beta-1,4$-endoglucanase in the root-knot nematode Meloidogyne incognita and expression analysis during plant parasitism. Mol. Plant-Microbe Interact. 12:585-591.

Rouppe van der Voort, J. N. A. M., van Zandvoort. P., Overmars, H., Helder, J., and Bakker, J. 1999. Linkage analysis by genotyping of sibling populations: a genetic map for the potato cyst nematode constructed using a "pseudo-F2" mapping strategy. Mol. Gen. Genet. 261:1021-1031.

Schots, A., de Boer, J. M., Schouten, A., Roosien, J., Zilverentant, J. F., Pomp, H., Bouwman, L., Overmars, H., Gommers, F. J., Visser, B., Stiekema, W. J., and Bakker, J. 1992. "Plantibodies": A flexible approach to design resistance against pathogens. Neth. J. Plant Pathol. Suppl. 2:183-191.

Smant, G., Goverse, A., Stokkermans, J. P. W. G., de Boer, J. M., Pomp, H. R., Zilverentant, J. F., Overmars, H. A., Helder, J., Schots, A., and Bakker, J. 1997. Potato root diffusate-induced secretion of soluble, basic proteins originating from the subventral esophageal glands of potato cyst nematodes. Phytopathology 87:839-845.

Smant, G., Stokkermans, J. P., Yan, Y., de Boer, J., Baum, T. J., Wang, X., Hussey, R. S., Gommers, F. J., Henrissat, B., Davis, E. L., Helder, J., Schots, A., and Bakker, J. 1998. Endogenous cellulases in animals: isolation of beta-1, 4-endoglucanase genes from two species of plantparasitic cyst nematodes. Proc. Natl. Acad. Sci. USA 95:4906-11

Tabara, H., Motohashi, T., and Kohara, Y. 1996. A multi-well version of in situ hybridization on whole mount embryos of Caenorhabditis elegans. Nucleic Acids Res. 24:2119-2124.

Vos, P., Hogers, R., Bleeker, M., Reijans, M., van-de, L. T., Hornes, M. Frijters, A., Pot, J., Peleman, J., Kuiper, M., and Zabeau, M. 1995. AFLP: A new technique for DNA fingerprinting. Nucleic Acids Res. 23:4407-4414.

Williamson, V. M., and Hussey, R. S. 1996. Nematode pathogenesis and resistance in plants. Plant Cell 8:1735-1745.

Wyss, U. 1992. Observations on the feeding behaviour of Heterodera schachtii throughout development, including events during moulting. Fundam. Appl. Nematol. 15:75-89.

Wyss, U., and Zunke, U. 1986. Observations on the behaviour of second stage juveniles of Heterodera schachtii inside host roots. Rev. Nematol. 9:153-165.

Yan, Y., Smant, G., Stokkermans, J., Qin, L., Helder, J., Baum, T., Schots, A., and Davis, E. L. 1998. Genomic organization of four beta1,4-endoglucanase genes in plant-parasitic cyst nematodes and its evolutionary implications. Gene 220:61-70. 